\title{
Changes in Electrical Activity of the Diaphragm in Response to Painful Procedures in Neonates
}

Daniel Lubarsky, BS, Kimberly S. Firestone, MSc, RRT, Ram K. Mukherjee, MS, Howard M. Stein, MD

\begin{abstract}
Background: Premature neonates are exposed to numerous painful procedures. Physiologic fluctuations in heart rate (HR), respiratory rate, and oxygen saturation are typically used to determine the response to pain. Neurally assisted ventilatory assist (NAVA) delivers inspiratory pressure in proportion to the electrical activity of the diaphragm (Edi). Since NAVA allows self-regulation of peak inspiratory pressures (PIP), there is apprehension that painful stimulus may increase respiratory drive and result in excessive PIP. This study evaluated changes in respiratory drive, measured by Edi, in response to a painful procedure (heel stick) to determine if there was excessive PIP delivered from the ventilator.

Methods: Prospective, single-center study; subjects $<32$ weeks on NAVA/NIV NAVA requiring routine blood work via heel sticks. Vital signs were measured every 10 seconds for the first 2 minutes. PIP, Edi peak, and min were collected for the first ten breaths and then averaged at 1 and 2 minutes. Statistics were repeated measures ANOVA.

Results: Fourteen subjects with gestational age 26.9+2 weeks and birth weight 994+318 grams. At study, the average age was $4.6+5$ days, and weight was $948+305$ grams. Following the heel stick, the first breath showed an increase in PIP and Edi peak but returned to baseline by the second breath. PIP increased again by the 10th breath and at 1 and 2 minutes. HR increased after heel stick and remained elevated through the remainder of the study. There were no changes in tonic Edi, Edi min or other vital signs. Conclusion: Although the increase in PIP and Edi peak in response to heel stick pain was brief and limited, it is important always to set the PIP alarm limit appropriately to protect the lung from excessive pressures that may be generated during painful procedures.
\end{abstract}

Keywords: Neurally Adjust Ventilatory Assist (NAVA), Pain, Premature Infants, Neural trigger. Peak Inspiratory Pressure.

Introduction:

Premature neonates experience recurrent painful procedures during their treatment course in the Neonatal Intensive Care Unit. Repeated invasive procedures occur routinely in these neonates, causing pain at a time when it is developmentally unexpected. (1) They can experience an average of 141 procedures during hospitalization with an average of 16 procedures per day, predominantly heel sticks. (2) Heel sticks have been shown to be more painful compared to traditional venous blood sampling (3) The neonatal physiologic response to pain consists of changes in endocrine (cortisol and catecholamine release), autonomic (increases in heart rate $(H R)$, respiratory rate $(R R)$, blood pressure, oxygen saturation (Sat)), and/or behavioral responses (facial action, body movement, and cry). $(1,4,5)$ Neonates are hypersensitive to pain and touch, and their behavioral response is altered compared to older infants. $(6,7)$ Although both facial responses and body movement increase with gestational age, neonates at younger gestational ages display more body movement and less facial responses. $(4,8)$ Endocrine responses to pain, especially catecholamine release, contribute to the autonomic pain response observed in neonates. This is evident through increases in HR and blood pressure Sat and RR. (5,9-13)

\section{"It is, therefore, possible that an increase in the respiratory drive from a non- respiratory cause (such as pain) may result in excessively high Edi signals and subsequent inappropriate PIP delivery while on NAVA."}

Despite alterations in RR and Sat, it is unknown what effect painful stimuli have on the respiratory drive. It is possible to measure the respiratory drive through the electrical activity of the diaphragm (Edi). Neurally adjusted ventilatory assist (NAVA) uses the respiratory drive (Edi peak) and tonic activity of the diaphragm (Edi $\mathrm{min}$ ) to provide synchronized proportional assist ventilation. (14) A specialized nasogastric catheter is positioned at the level of the diaphragm, and embedded electrodes continuously detect the Edi. The instantaneous tonic Edi (Edi peak - min) is multiplied by a proportionality constant, the NAVA level, to calculate the delivered peak inspiratory pressure (PIP) every 16 milliseconds. Patients can, therefore, determine the amount of PIP delivered by the ventilator, breath to breath, by varying the size of the Edi signal. (15) Various authors have expressed apprehensions about premature neonates' ability to safely and appropriately direct their own ventilation.(16-18) It is, therefore, possible that an increase in the respiratory drive from a non-respiratory cause (such as pain) may result in excessively high Edi signals and subsequent inappropriate PIP delivery while on NAVA.

The purpose of this study was to correlate the change in respiratory drive, as measured by Edi peak, in response to a painful procedure, such as a heel stick, in preterm neonates.

\section{Methods:}

This was a prospective, single-center observational study. The study size was a convenience sample enrolling subjects $<32$ weeks on NIV NAVA (Servo-I ventilator, Getinge, Germany) who required routine blood work via heel sticks. IRB approval and informed consent were obtained. Exclusion criteria for this study

NEONATOLOGY TODAY is interested in publishing manuscripts from Neonatologists, Fellows, NNPs and those involved in caring for neonates on case studies, research results, hospital news, meeting announcements, and other pertinent topics. Please submit your manuscript to: LomaLindaPublishingCompany@gmail.com 
included the use of analgesia, sedation, or the presence of brain injury (HIE, IVH grade 3 \& 4).

Baseline measurements were taken 5 minutes before the heel stick prior to the subject being disturbed. HR, Sat, and RR were measured every 10 seconds for the first 2 minutes after the heel stick. PIP, Edi peak, and min were recorded and downloaded from the ventilator 5 minutes before the heel stick as a baseline, at the time of the heel stick, for the first ten breaths after the heel stick, and then averaged over a minute at 1 minute and at 2 minutes. Tonic Edi was calculated (Edi peak minus Edi min) as this is how the ventilator calculates the amount of PIP to deliver.

\section{Statistics:}

The dependent variables were HR, Sat, RR, PIP, Edi peak and min, and Tonic Edi. The independent variable was the heel stick event. The examination of data included the calculation of summary statistics for continuous data for each group. Based on the clinical rationale, each observation was viewed as independent; therefore, the statistical analysis was conducted on the full set of observations. The repeated measure ANOVA was conducted to assess if there is any difference across time points for each of the groups and, if found, a post hoc analysis, using Bonferroni correction, was carried out to check which time point was significantly different to the baseline. Data were log-transformed to achieve better normality and constant variance. Statistical analyses were completed using "Imertest" package of R-version R-3.4.4 (GNU General Public License, Free Software Foundation, Inc.). All testing was two-tailed and evaluated at the Type I Error Rate of al- pha $=0.05$ level of statistical significance.

\section{Results:}

Fourteen study subjects (10 females) were enrolled. The gestational age was 26.9 \pm 2 weeks (range 24-31 weeks), and birth weight was $994 \pm 318$ grams (range $500-1340$ grams). At the time of the study, the age was $4.6 \pm 5$ days (range $0-17$ days), and weight was $948+305$ grams (range $450-1690$ grams). All subjects received prenatal steroids, and $93 \%$ received surfactant. Median Apgar scores were 4 and 7 at 1 and 5 minutes, respectively. Median ventilator settings at the time of the studies were: NAVA level of 1 (range $0-2$ ) $\mathrm{cmH}_{2} \mathrm{O} / \mathrm{mcV}$, peak pressure limit 35 (range 3035) $\mathrm{cmH}_{2} \mathrm{O}$, apnea time 2 seconds, peep 8 (range 6-10) $\mathrm{cmH}_{2} \mathrm{O}$, backup pressure control 20 (range 18-23) $\mathrm{cmH}_{2} \mathrm{O}, \mathrm{RR} 40$ (range 30-50) breaths per minute. All subjects were in various stages of resolving RDS, treated with caffeine, and used the RAM cannula as the interface system (Neotech, California).

Table 1 shows the repeated measure ANOVA which demonstrated significant difference over time for Edi peak and PIP $(p<0.05)$ but no changes for tonic Edi or Edi min. Tables 2 and 3 show the post hoc analysis, using Bonferroni correction, to determine which time points were different from baseline for Edi peak and PIP. Both Edi peak and PIP showed significant increases with the first breath after the heel stick, but only PIP continued to show these increases at the $10^{\text {th }}$ breath and at 1 and 2 minutes after the heel stick.

Figure 1 demonstrates the breath-to-breath responses to the heel stick for PIP, tonic Edi, and Edi peak and min. The data was not normally distributed, so it is shown as mean, median, interquar-

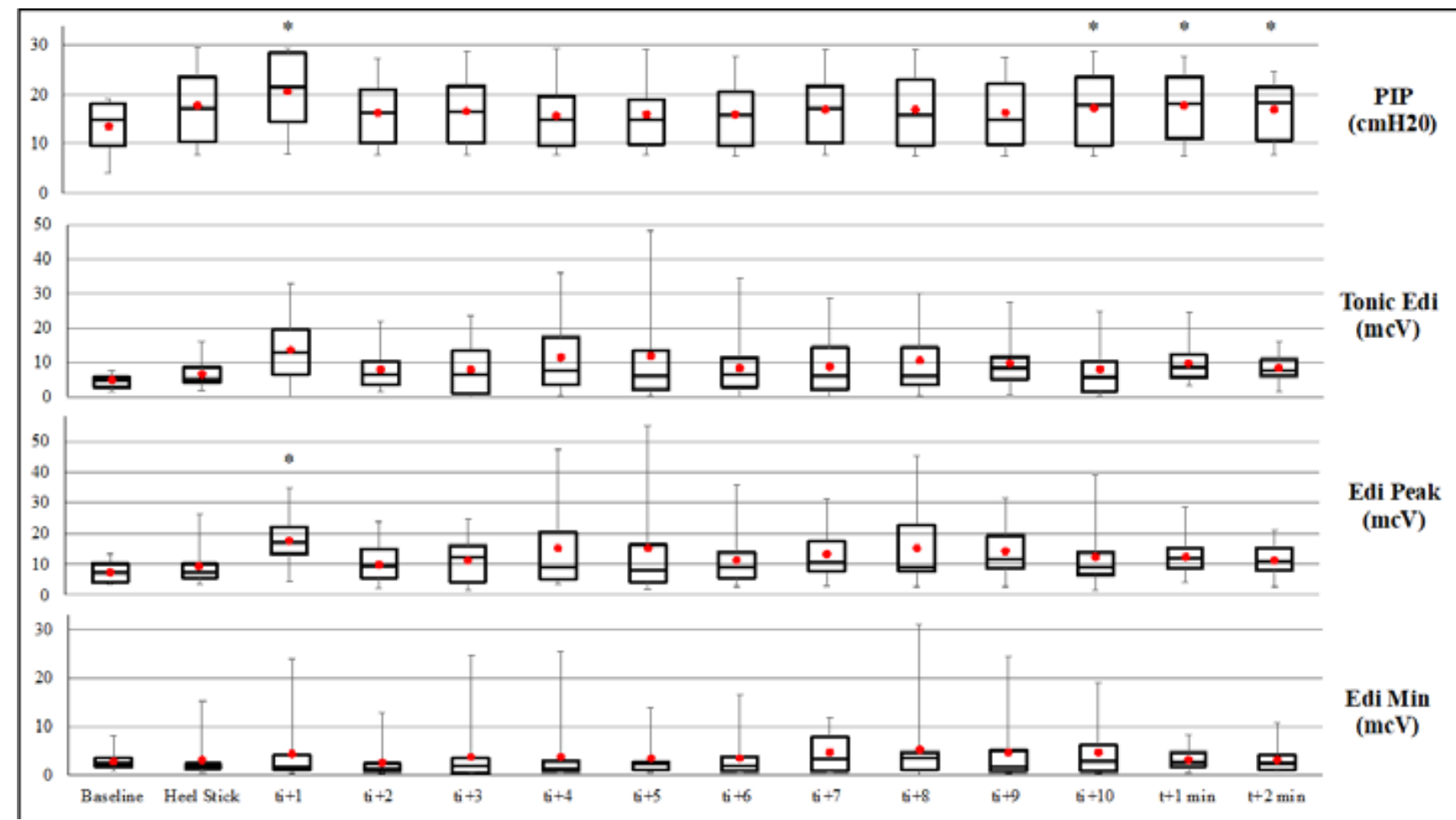

Figure 1: Respiratory response to pain (heel stick). The first 10 breaths after the heel stick are designated as ti+1 to ti+10, $t+1$ and $t+5$ min refers to 1 and 5 minutes after the heel stick. The red solid circles are the mean values. The boxplots show the median and first and third quartiles. The whiskers are the minimum and maximum values. The first breath following the heel increased for PIP and Edi peak followed by a return to baseline. PIP increased again at breath 10 and at 1 and 2 minutes. There were no changes in tonic Edi and Edi min throughout the study. ${ }^{*}=p<0.05$ compared to baseline. 


\begin{tabular}{|l|l|l|l|l|l|l|}
\hline & $\begin{array}{l}\text { Sum of } \\
\text { Squares }\end{array}$ & $\begin{array}{l}\text { Mean } \\
\text { Sum of } \\
\text { Sq. }\end{array}$ & $\begin{array}{l}\text { Numerator } \\
\text { DF }\end{array}$ & $\begin{array}{l}\text { Denominator } \\
\text { DF }\end{array}$ & F Value & P-value \\
\hline PIP & 1.4906 & 0.12421 & 12 & 156 & 2.9596 & 0.000969 \\
\hline Edi Peak & 7.3157 & 0.60964 & 12 & 156 & 1.9744 & 0.0299 \\
\hline Edi Min & 6.3877 & 0.53231 & 12 & 156 & 0.8154 & 0.6343 \\
\hline Tonic Edi & 6.6484 & 0.55403 & 12 & 156 & 1.0767 & 0.3833 \\
\hline
\end{tabular}

Table 1: Repeated Measure ANOVA results. Type III Analysis of Variance Table with Satterthwaite's method. Only $P I P$ and Edi peak showed significant differences over the study period $(p<0.05)$.

\begin{tabular}{|l|l|l|l|l|l|l|l|}
\hline Edi Peak & Estimate & $\begin{array}{l}\text { Standard } \\
\text { Error }\end{array}$ & Z Value & P-value & $\begin{array}{l}\text { CI } \\
\text { Estimate }\end{array}$ & Lower & Upper \\
\hline Baseline-ti+1 & -0.8295 & 0.21 & -3.95 & $\mathbf{0 . 0 0 0 9 4}$ & 0.436 & 0.244 & 0.78 \\
\hline Baseline-ti+2 & -0.1977 & 0.21 & -0.941 & 1.00 & 0.821 & 0.459 & 1.467 \\
\hline Baseline-ti+3 & -0.1702 & 0.21 & -0.811 & 1.00 & 0.843 & 0.472 & 1.508 \\
\hline Baseline-ti+4 & -0.4504 & 0.21 & -2.144 & 0.3841 & 0.637 & 0.356 & 1.14 \\
\hline Baseline-ti+5 & -0.2784 & 0.21 & -1.326 & 1.00 & 0.757 & 0.423 & 1.354 \\
\hline Baseline-ti+6 & -0.2552 & 0.21 & -1.215 & 1.00 & 0.775 & 0.433 & 1.385 \\
\hline Baseline-ti+7 & -0.4624 & 0.21 & -2.202 & 0.3323 & 0.63 & 0.352 & 1.126 \\
\hline Baseline-ti+8 & -0.5167 & 0.21 & -2.460 & 0.1666 & 0.596 & 0.334 & 1.067 \\
\hline Baseline-ti+9 & -0.5082 & 0.21 & -2.420 & 0.1864 & 0.602 & 0.336 & 1.076 \\
\hline Baseline-ti+10 & -0.29 & 0.21 & -1.381 & 1.00 & 0.748 & 0.418 & 1.338 \\
\hline Baseline-t+1 min & -0.5093 & 0.21 & -2.425 & 0.1838 & 0.601 & 0.336 & 1.075 \\
\hline Baseline- $\mathrm{t}+2$ min & -0.4185 & 0.21 & -1.993 & 0.5558 & 0.658 & 0.368 & 1.177 \\
\hline
\end{tabular}

Table 2: Post hoc analysis for Edi peak using Bonferroni correction. Significant increases are bolded

\begin{tabular}{|l|l|l|l|l|l|l|l|}
\hline PIP & Estimate & $\begin{array}{l}\text { Standard } \\
\text { Error }\end{array}$ & Z Value & P-value & $\begin{array}{l}\text { CI } \\
\text { Estimate }\end{array}$ & Lower & Upper \\
\hline Baseline-ti+1 & -0.42975 & 0.07743 & -5.55 & $\mathbf{3 . 4 3 e - 0 7}$ & 0.651 & 0.525 & 0.806 \\
\hline Baseline-ti+2 +-0.19987 & 0.07743 & -2.581 & 0.11816 & 0.819 & 0.661 & 1.015 \\
\hline Baseline-ti+3 & -0.20430 & 0.07743 & -2.638 & 0.09994 & 0.815 & 0.658 & 1.01 \\
\hline Baseline-ti+4 & -0.14637 & 0.07743 & -1.89 & 0.70458 & 0.864 & 0.697 & 1.07 \\
\hline Baseline-ti+5 & -0.16142 & 0.07743 & -2.085 & 0.44522 & 0.851 & 0.687 & 1.054 \\
\hline Baseline-ti+6 & -0.15687 & 0.07743 & -2.026 & 0.51339 & 0.855 & 0.69 & 1.059 \\
\hline Baseline-ti+7 & -0.2135 & 0.07743 & -2.757 & 0.06995 & 0.808 & 0.652 & 1.001 \\
\hline Baseline-ti+8 & -0.19953 & 0.07743 & -2.577 & 0.11966 & 0.819 & 0.661 & 1.015 \\
\hline Baseline-ti+9 & -0.16765 & 0.07743 & -2.165 & 0.36452 & 0.846 & 0.683 & 1.048 \\
\hline Baseline-ti+10 & -0.22639 & 0.07743 & -2.924 & $\mathbf{0 . 0 4 1 5 1}$ & 0.797 & 0.644 & 0.988 \\
\hline Baseline-t+1 min & -0.26334 & 0.07743 & -3.401 & $\mathbf{0 . 0 0 8 0 6}$ & 0.768 & 0.62 & 0.952 \\
\hline Baseline- $t+2$ min & -0.23884 & 0.07743 & -3.085 & $\mathbf{0 . 0 2 4 4 6}$ & 0.788 & 0.636 & 0.976 \\
\hline
\end{tabular}

Table 3: Post hoc analysis for PIP using Bonferroni correction. Significant increases are bolded 


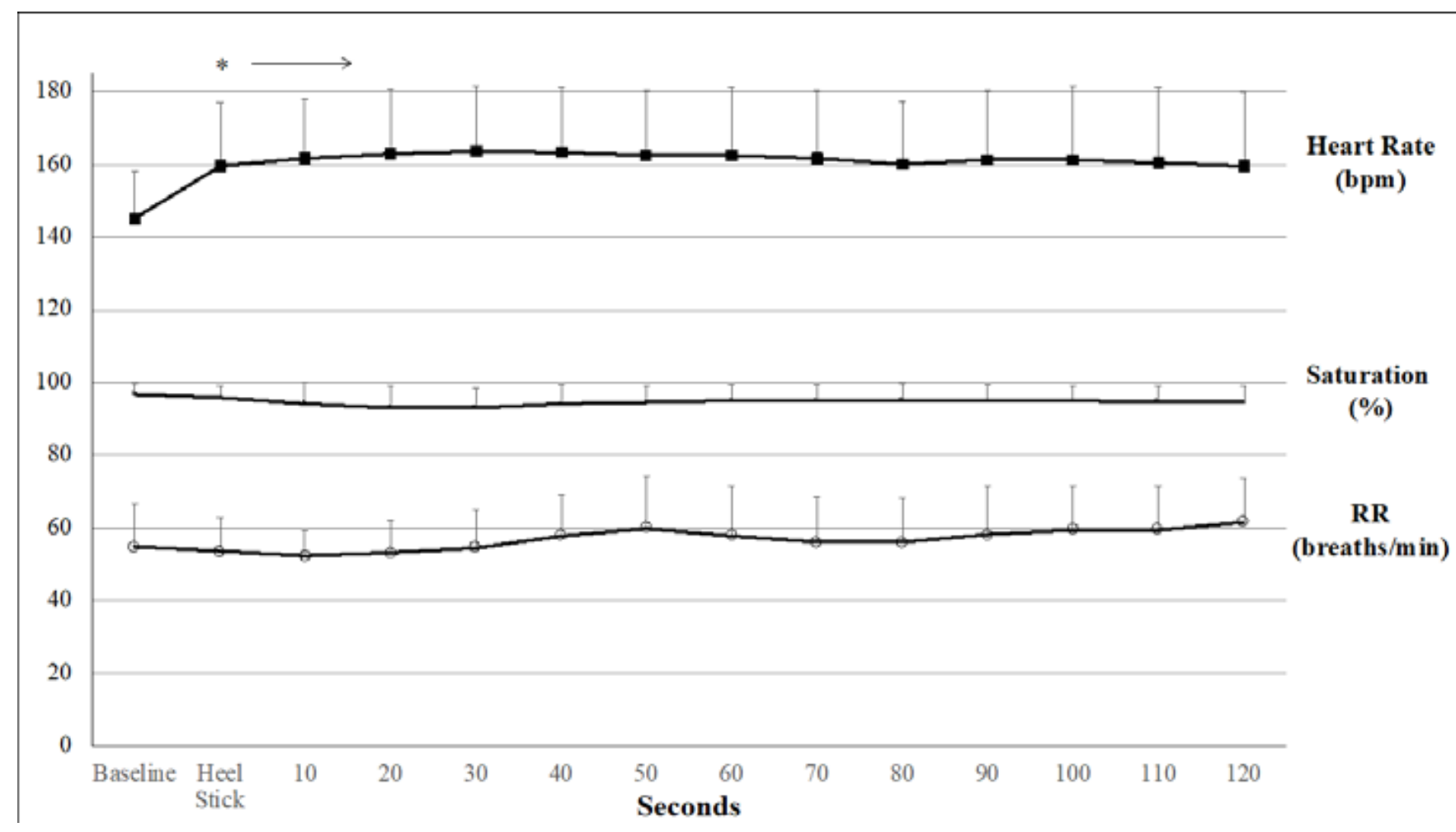

Figure 2: Response of RR (breaths/min), HR (bpm), and Sat (\%) to the heel stick stimuli. Following the heel stick, HR increased within 10 seconds from baseline and remained elevated for the remainder of the two minutes $\left({ }^{*}=p<0.05\right)$. There were no significant changes seen in either RR or Sat in response to the heel stick. Data were normally distributed and shown as mean $\pm S D$.

tile ranges, maximum and minimum. Following the heel stick, the first breath showed an immediate increase in both Edi peak and PIP, but both decreased by the second breath. Only PIP increased again by the tenth breath and at 1 and 2 minutes after the painful stimulus. There were no significant changes in tonic Edi or Edi min throughout the study.

Vital sign response to the heel stick is shown in Figure 2. Following the heel stick, HR immediately increased and remained elevated for the remainder of the study. There were no changes in either RR or Sat in response to the heel stick.

Although there were some increases in PIP and Edi peak, the majority of the values were within the acceptable clinical range. However, there were some extreme values, as noted by the maximum values. Because of the potential harm from excessive PIP, the percent increase of the maximal value from the mean baseline of each variable was calculated. Figure 3 demonstrates the percent increase above baseline for these extreme values. Although tonic Edi, Edi peak and min had percent increases up to $900 \%$ over baseline, the peak pressure limit (set at $35 \mathrm{cmH}_{2} \mathrm{O}$ to limit the PIP to $30 \mathrm{cmH}_{2} \mathrm{O}$ ) restricted PIP increases to just over $100 \%$ increase from mean baseline PIP (13.5 $\mathrm{cmH} 2 \mathrm{O})$.

\section{Discussion:}

This is the first study to examine changes in respiratory drive, as measured by Edi, and PIP in response to a painful stimulus (heel stick) in premature neonates. There was an increase in the Edi peak and PIP with the first breath after the painful stimulus and then a return to baseline until PIP increased again at 1 and 2 minutes after the heel stick. The neonates also responded to the painful stimulus with an increased HR consistent with previous reports of physiological responses to pain. $(10,11,13,19,20)$ There was, however, no increase in the respiratory rate for the two minutes following the stimulus contrary to previously described pain responses in neonates. (11)

\section{"This is the first study to examine changes in respiratory drive, as measured by Edi, and PIP in response to a painful stimulus (heel stick) in premature neonates."}

We chose to look at the first ten breaths because previous bedside observations suggested that Edi responded rapidly within 1-2 breaths to various stimuli. We chose to record the variables out to 2 minutes because the painful part of the procedure was complete, and the neonate most likely had returned to baseline. However, both heart rate and PIP continued to be increased at the end of the study.

The increase in HR suggests that neonates responded to the heel stick with catecholamine release and resultant tachycardia. Neonates on ventilators, or with increased illness severity scores or prior painful procedures, were perceived to have lower pain intensity scores and may explain why there was no increase in RR or Sat. $(21,22)$ In addition, premature infants exhibit less behavioral responses to pain with younger postmenstrual age at birth, lower birthweight, mechanical ventilation, and longer length of stay in the NICU. The behavioral indicators that typically increased dur- 


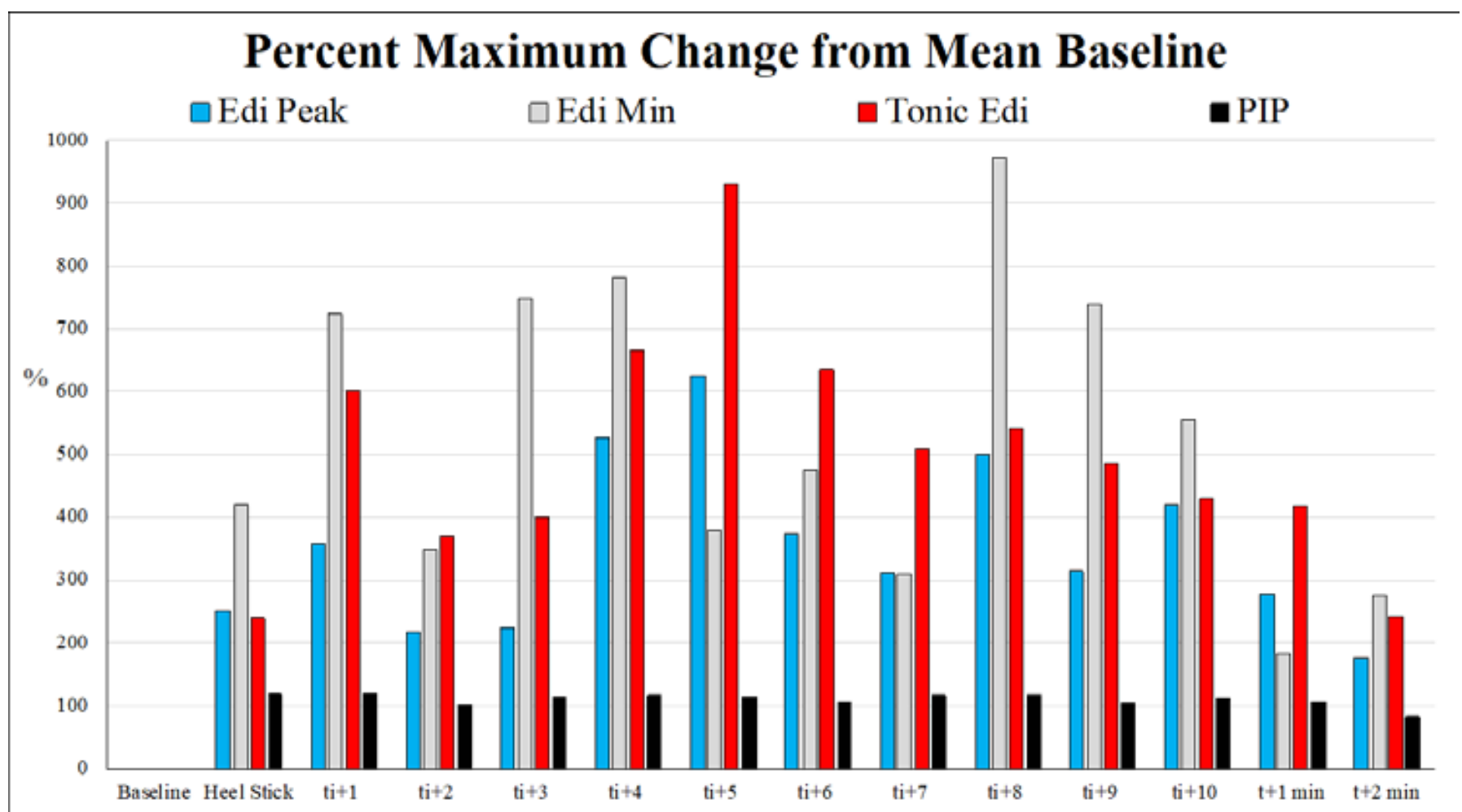

Figure 3: Percent maximum increase from mean baseline for PIP, tonic Edi, Edi peak and min. Despite large increases in tonic Edi, the peak pressure limit prevented excessive PIP increases.

ing heel stick procedure, including crying, arousal state, and facial grimace and were not measured in this study. (23)

NAVA allows self-regulation of PIP raising the concern that painful stimuli may increase respiratory drive and result in excessive PIP being delivered to the neonate. This study showed that there was an increase in Edi peak and the resultant increase in PIP, but this lasted for one breath only and then decreased back to baseline. The sustained increase in HR and the increased PIP at 1 and 2 minutes after the heel stick most likely reflects ongoing stimuli from the bedside provider completing the blood collection from the heel stick.

Although PIP did have increases over the study period, the mean, median, and upper quartile values were all within clinically acceptable ranges, especially for non-invasive ventilation. The ventilator determines the amount of PIP to deliver based on the instantaneous tonic Edi (Edi peak - min). Therefore, large increases in Edi peak and min can result in normal tonic Edi but can also result in excessively high values. Edi peak increase was above the normal Edi peak ranges of $5-15 \mathrm{mcV}$ noted for premature neonates but well within previously observed normal ranges, (24) Edi min were variable and, at times, were significantly elevated. To protect the neonate from excessively high PIP, the ventilator has a peak pressure limit that controls the maximum amount of PIP delivered. The peak pressure limit, found in the alarm screen of the Servo I ventilator, is the essential setting to protect the lung from potential baro or volu-trauma but still allow recruitment of lung for effective ventilation when needed. (15). If this pressure limit is set appropriately during physiologic ventilation, any excessive increase in Edi secondary to the response to pain will result in the pressure popping off $5 \mathrm{cmH}_{2} \mathrm{O}$ below the set pressure limit and should protect the lung from excessive pressures. This was shown in Figure 3 , where there were 3-10 fold increase in maximum tonic Edi but only a two fold increase in maximum PIP. Setting the peak limit at $35 \mathrm{cmH}_{2} \mathrm{O}$ limited the maximum PIP to $30 \mathrm{cmH}_{2} \mathrm{O}$.

This study was limited by the inability to control for variations in bedside comforting techniques while administering heel sticks, although the NICU nurses were all taught similar calming techniques. In addition, pain from the heel stick itself could not be differentiated from the response from being stimulated and/or the heel being squeezed during the subsequent blood draw. However, of practical relevance, none of the above issues resulted in sustained increases PIP, most likely due to the appropriate setting of the peak pressure limit. Future studies would include evaluating the effectiveness of various pain mitigation techniques on the Edi response.

\section{"This study adds to the safety profile of NAVA by suggesting that although premature neonates respond to pain with increases in their respiratory drive, these increases do not trigger sustained, excessive PIPs,"}

\section{Conclusion:}

NAVA ventilation is gaining wider acceptance as a mode of ventilation in neonates, but apprehension exists concerning the premature neonates' ability to safely and appropriately direct their own ventilation.(16-18) This study adds to the safety profile of NAVA 
by suggesting that although premature neonates respond to pain with increases in their respiratory drive, these increases do not trigger sustained, excessive PIPs. Bedside clinical management of neonates on NIV NAVA must always include appropriate safety settings by setting the pressure limit high enough during physiologic ventilation to allow adequate lung recruitment while protecting the lungs from excessive PIP.

Acknowledgments: We thank Deepika Murala Kennedy for help with the original study proposal and data collection.

\section{References:}

1. Anand K, Aranda J, Berde C, et al. Summary proceedings from the neonatal pain-control group. Pediatrics. 2006;117(3 Pt 2):S9-S22.

2. Carbajal R, Rousset $A$, Danan $C$, et al. Epidemiology and treatment of painful procedures in neonates in intensive care units. JAMA. 2008;300(1):60-70.

3. Shah V, Taddio A, Bennett S, Speidel B. Neonatal pain response to heel stick vs. venepuncture for routine blood sampling. Arch Dis Child. 1997;77:F143-144.

4. Craig K, Whitfield M, Grunau R, Linton J, Hadjistavropoulos $H$. Pain in the preterm neonate: behavioural and physiological indices. Pain. 1993;52(3):287-299.

5. Mclntosh N, Van Veen L, Brameyer H. The pain of heel prick and its measurement in preterm infants. Pain. 1993;52:71-74.

6. Andrews K, Fitzgerald $M$. The cutaneous withdrawal reflex in human neonates: sensitization, receptive fields, and the effects of contralateral stimulation. Pain. 1994;56:95-101.

7. Fitzgerald M, Millard C, Mclntosh N. Cutaneous hypersensitivity following peripheral tissue damage in newborn infants and its reversal with topical anaesthesia. Pain. 1989;39(1):31-36.

8. Morison S, Holsti L, Grunau R, et al. Are there developmentally distinct motor indicators of pain in preterm infants? . Early Hum Dev. 2003;72(2):131-146.

9. Ranger M, Johnston C, Anand K. Current Controversies Regarding Pain Assessment in Neonates. Semin Perinatol. 2007;31(5):283-288.

10. Borgbjerg F, Nielsen K, Franks J. Experimental pain stimulates respiration and attenuates morphine-induced respiratory depression: a controlled study in human volunteers. Pain. 1996;64(1):123-128.

11. Bourke $D$. Respiratory effects of regional anesthesia during acute pain. Reg Anesth. 1993;18(6):361-365.

12. Rawlings $D$, Miller $P$, Engel R. The Effect of Circumcision on Transcutaneous PO2 in Term Infants. Am J Dis Child. 1980;134:676-678.

13. Brown L. Physiologic responses to cutaneous pain in neonates. Neonatal Network. 1987;5:18-23.

14. Sinderby C, Beck J. "Neurally Adjusted Ventilatory Assist". Principles and Practice of Mechanical Ventilation. Third ed: McGraw Hill; 2012.

15. Firestone KS, Beck J, Stein H. Neurally Adjusted Ventilatory Assist for non-invasive support in neonates. Clin Perinatol. 2016;43(4):707-724.

16. DiBlasi RM. The Importance of Synchronization During Neonatal Noninvasive Ventilation Resp Care. 2018;63(12):1579-1582.

17. Rossor T, Hunt K, Shetty A, Greenough A. Neurally Adjusted ventilatory assist compared to other forms of triggered ventilation for neonatal respiratory support. Cochrane database of systemic reviews. 2017;10.

18. Turner D, Rehder K, Cheifitz I. Nontraditional modes of mechanical ventilation: progress or distraction? Expert Rev of Respir Med. 2012;6(3):277-284.
19. Pineles B, Sandman C, Waffarn F, Uy C, Poggi E. Sensitization of Cardiac Responses to Pain in Preterm Infants. Neonatology. 2007;91:190-195.

20. Worley A, Fabrizi L, Boyd S, Slater R. Multi-modal pain measurements in infants. $J$ Neurosci Methods. 2012;205(2):252-257.

21. Grunau $R$, Oberlander $T$, Whitfield $M$, Fitzgerald $C$, Lee $S$. Demographic and therapeutic determinants of pain reactivity in very low birth weight neonates at 32 weeks' postconceptional age. Pediatrics. 2001;107:105-112.

22. Peters J, Koot $H$, Grunau $R$, et al. Neonatal facial coding system for assessing postoperative pain in infants: item reduction is valid and feasible. Clin J Pain. 2003;19(6):353363.

23. Williams A, Khattak A, Garza C, Lasky R. The behavioral pain response to heelstick in preterm neonates studied longitudinally: Description, development, determinants, and components. Early Hum Dev. 20019;85:369-374.

24. Stein $H$, Hall $R$, Davis $K$, White DB. Electrical activity of the diaphragm (Edi) values and Edi catheter placement in nonventilated preterm neonates. J Perinatol. 2013;33(9):707711.

Disclosures:

HS and KF are on the speakers' bureau for Getinge and Chiesi.

$D L$ and RM have no conflicts to disclose.

HS and KF contributed to study design, data collection, analysis and writing the manuscript.

$D L$ and RM contributed to data analysis and writing the manuscript.
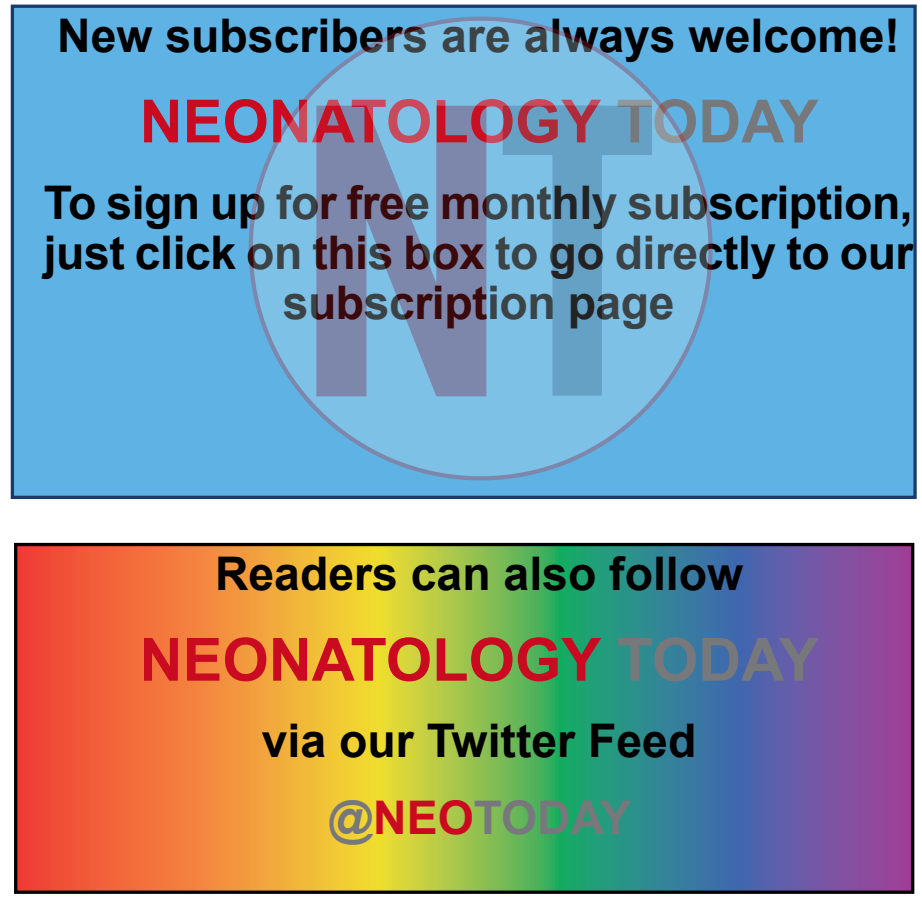


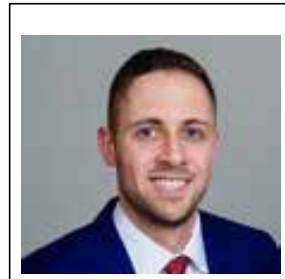

Daniel Lubarsky, BS

$3^{\text {rd }}$ year medical student

University of Toledo College of Medicine

Toledo, Ohio

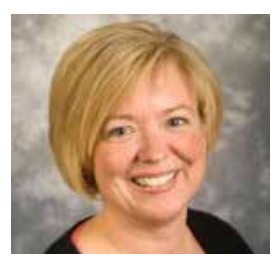

Kimberly S. Firestone MSc, RRT

Neonatal Outreach Cordinator

Akron Children's Hospital

Akron, Ohio
Principle Author

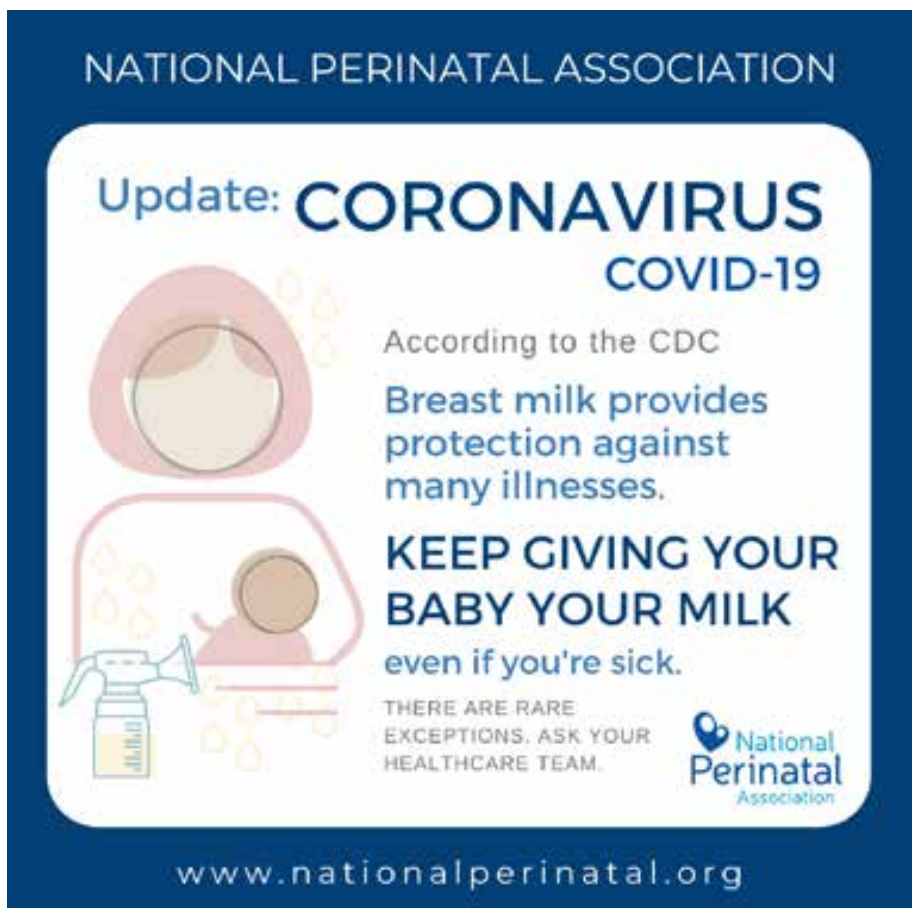

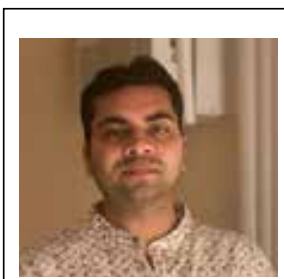

Ram Mukherjee, MS

PhD candidate, Department of Mathematics and Statistics,

University of Toledo

Toledo, Ohio

\section{Corresponding Author}

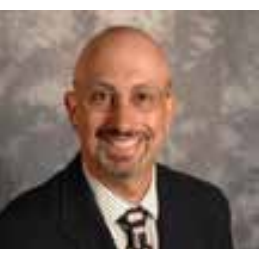

Howard Stein, MD

Medical Director, Neonatology

ProMedica Ebeid Children's Hospital, Toledo, Ohio

Professor of Pediatrics, University of Toledo College of Medicine and Life Sciences,

Toledo, Ohio

Howardstein@bex.net

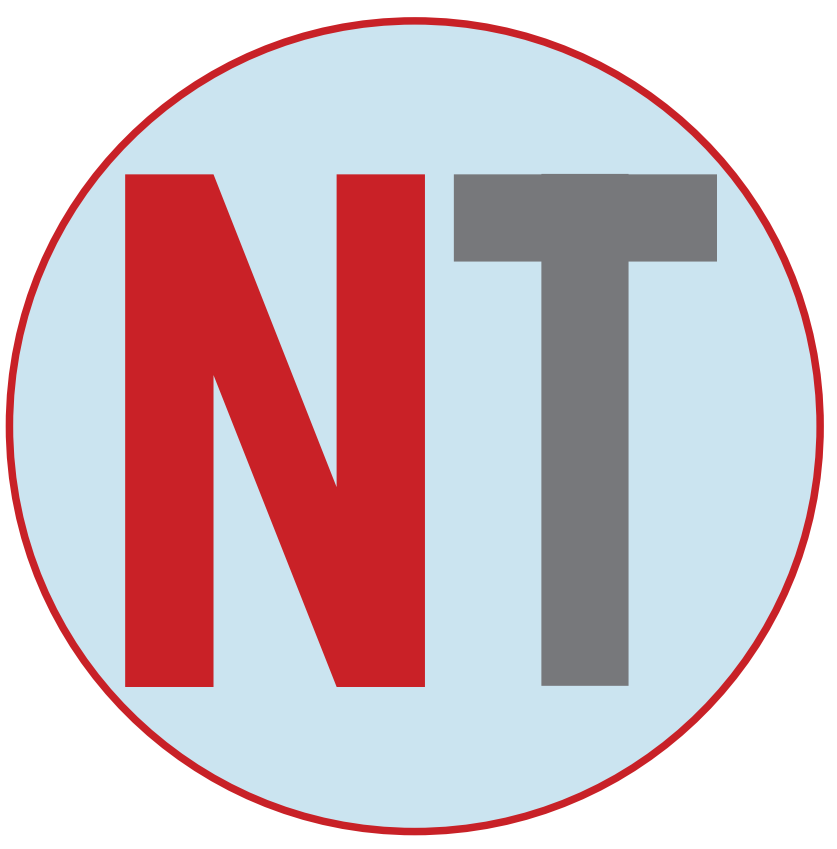

